2018-06

\title{
Eye movement attenuation of intrusive social anxiety imagery: A pilot study.
}

\author{
Homer, Sophie
}

http://hdl.handle.net/10026.1/10484

10.1016/j.jbtep.2017.11.003

J Behav Ther Exp Psychiatry

Elsevier

All content in PEARL is protected by copyright law. Author manuscripts are made available in accordance with publisher policies. Please cite only the published version using the details provided on the item record or document. In the absence of an open licence (e.g. Creative Commons), permissions for further reuse of content should be sought from the publisher or author. 
Eye movement attenuation of intrusive social anxiety imagery: A pilot study.

\section{Sophie R. Homer \& Catherine Deeprose}

School of Psychology, Cognition Institute, Plymouth University, Drake Circus, Plymouth, Devon, PL4 8AA, UNITED KINGDOM

${ }^{*}$ Corresponding author

E-mail address: $\underline{\text { sophie.homer@plymouth.ac.uk }}$

Postal address:

Sophie Homer,

Link 301,

Plymouth University,

Drake Circus,

Plymouth,

Devon,

PL4 8AA

UNITED KINGDOM 


\section{Highlights}

- Eye movements (EMs) are applied to involuntary and intrusive social anxiety imagery

- Images were lower in vividness after EMs than after a control task

- EMs may prevent increases in intrusive image vividness following exposure

\section{Abstract}

Background and objectives: A growing body of research has shown that negative, intrusive mental imagery plays a prevalent and causal role in social anxiety, and is qualitatively different to voluntarily generated imagery. Negative imagery can be reduced in vividness and emotional intensity through concurrent lateral eye movements (EMs) as per Eye Movement Desensitisation and Reprocessing (EMDR) therapy. Despite EMDR being used for a range of disorders, previous laboratorybased experimental research typically uses voluntarily generated autobiographical memories rather than involuntarily experienced intrusive images. In a pilot study, we investigated the efficacy of eye movement attenuation of negative, intrusive, socialanxiety imagery.

Methods: Twenty-seven participants (aged 18-29, 20 females) screened for social anxiety using the Social Interaction Anxiety Scale and who experienced intrusive imagery visualised their image while making eye movements or completing a control task. Self-report ratings of image vividness and emotionality were taken at baseline, immediately after the tasks, and following a post-test visualisation of their image. 
Results: Vividness reduced from baseline during the eye movements task, but not the control task, and vividness was lower at post-test in the EMs condition than in the control condition. This effect was not observed for emotionality.

Limitations: As a pilot study, the sample size was small and so replication on a larger scale is warranted.

Conclusions: EMs may prevent increases in vividness as a result of exposure to intrusive imagery. These findings tentatively suggest a promising extension of the topical eye movements paradigm to intrusive social anxiety images, and potential justification for EMDR intervention outside of PTSD.

Key words: EMDR; eye movements; working memory; social anxiety; mental imagery; intrusive imagery 


\section{Introduction}

Recurrent and intrusive mental imagery based on negative autobiographical memory is a cardinal feature of Post-Traumatic Stress Disorder (PTSD; Hackmann, Ehlers, Speckens \& Clark, 2004; Holmes, Grey \& Young, 2004), but interest in its role as a transdiagnostic phenomenon is increasing (Brewin, Gregory, Lipton \& Burgess, 2010; Clark, James, lyadurai \& Holmes, 2015; Hackmann \& Holmes, 2004). In primarily adult, Western, and clinical/sub-clinical samples, qualitative investigations have shown its prevalence in depression, anxiety disorders, and psychosis, amongst other diagnoses (see Brewin et al., 2010 for a review). Accordingly, imagery focused interventions are receiving a growing amount of research attention. Eye Movement Desensitisation and Reprocessing Therapy (EMDR) is an evidence-based and NICE recommended therapy for PTSD (Bisson et al., 2007; Cloitre, 2009; Lee \& Cuijpers, 2013; NICE, 2005). Perhaps the most unique and controversial component of EMDR is its use of horizontal eye movements (EMs), during which clients visualise their trauma image with the aim of reducing its potency. Assessment of the efficacy of EMs in this regard, and attempts to determine their specific underlying mechanisms, have attracted a large body of basic science research. Typically, tasks requiring horizontal saccades are employed during the visualisation of negative autobiographical memories with pre-test and post-test ratings of self-reported image vividness and emotional intensity (emotionality) (see Jeffries \& Davis, 2013 and van den Hout \& Engelhard, 2012, for reviews). Investigated in this way, EMs have been shown most consistently to reduce the vividness, but at times also the emotionality of negative memories (e.g. Andrade, Kavanagh and Baddeley, 1997; Barrowcliff, Gray, Freeman \& MacCulloch, 2004; Kavanagh, Freese, Andrade \& May, 2001; Lilley, Andrade, Turpin, Sabin-Farrell and Holmes, 2009; van den Hout, Muris, 
Salemink \& Kindt, 2001) negative prospective imagery (Engelhard, van den Hout, Janssen \& van der Beek, 2010; Engelhard, van den Hout et al., 2011; Engelhard, Sijbr, van den Hout \& Rutherford, 2011), food craving imagery (Kemps, Tiggemann, Woods \& Soekov, 2004; McClelland, Kemps \& Tiggemann, 2006) and public speaking imagery (Homer, Deeprose \& Andrade, 2016; Kearns \& Engelhard, 2015). The general consensus is that EMs during image visualisation compete for limited working memory (WM) resources thus reducing image vividness (van den Hout \& Engelhard, 2012; Stewart, Homer, Deeprose \& Andrade, in prep.). In their degraded form, images consume fewer WM resources when visualised, allowing more resources to be allocated to the therapeutic elements of EMDR. However, some debate remains as to whether this interference should be modality-specific, i.e. visuospatial interference such as EMs preferentially attenuates visual imagery (Andrade, Kavanagh \& Baddeley, 1997), or general, i.e. any WM interference task attenuates imagery of any modality (Gunter \& Bodner, 2008).

Intrusive mental imagery features in cognitive models of social anxiety (e.g. Clark \& Wells, 1995; Hofmann, 2007) and is prevalent in both clinical and sub-clinical samples (e.g. Hackmann, Clark \& McManus, 2000; Homer \& Deeprose, 2017) and causes anxiety, negative affect, decreased self-esteem, and poorer social performance (Hulme, Hirsch, \& Stopa, 2012; Moscovitch et al., 2011; Hirsch, Clark, Mathews \& Williams, 2003; Hirsch, Meynen \& Clark, 2004; see Ng et al., 2014 for a review). In line with research showing that voluntary and involuntary memories can be independent (Conway and Pleydell-Pearce, 2000; Ehlers and Clark, 2000; Brewin and Holmes, 2003), intrusive social anxiety imagery is phenomenologically different to voluntarily generated imagery (for example, it may comprise self-representations 
or prospective imagery rather than contextualised episodic memories) and is associated with higher levels of anxiety (Homer \& Deeprose, 2017). This study sought to extend the EMs paradigm to intrusive, involuntary imagery in a sub-clinical socially anxious sample, to yield stronger clinical implications than previous research using voluntarily-generated images in healthy samples. EMDR for PTSD has a strong experimental and clinical evidence base (NICE, 2005), but its efficacy in other disorders, including phobias and anxiety disorders, is largely anecdotal (van den Hout \& Engelhard, 2012, see also Stewart et al., in prep.). This study represents a first step towards experimental testing of EMs / WM interference interventions for intrusive imagery directly associated with disorders other than PTSD.

In a small pilot study, undergraduate students screened to be high in social anxiety and who reported intrusive social imagery were asked to visualise the image they experience recurrently and intrusively while performing EMs or a control task in which the eyes remained stationary. We hypothesised that:

1) The EMs task would cause images to reduce in vividness and emotionality more so than the control task.

2) Images in the EMs condition would remain lower in vividness and emotionality than those in the control condition during the post-test visualisation. 


\section{Method}

\subsection{Participants}

One hundred and ten Plymouth University undergraduate students signed up to the study and took part in screening. Fifty students met screening criteria and thirty-eight attended the study for course credit or small payment. Twenty-seven participants reported experiencing intrusive mental imagery during a parallel investigation into image phenomenology (see Homer \& Deeprose, 2017) and are reported here, mean age 20 years, SD $=2.51$, age range $=18-29,20$ females. Four participants $(14.81 \%)$ disclosed a diagnosis of anxiety, one participant (3.70\%) indicated uncertainty and two participants $(7.41 \%)$ declined to respond to this question.

\subsection{Measures}

Screening measure: Participants were screened using the Social Interaction Anxiety Scale (SIAS) (Mattick \& Clark, 1998). Participants endorsed statements such as "I have difficulty talking with other people" on a 5-point Likert scale scored 0-4 (never/almost never; not usually; sometimes; usually; always/almost always), providing scores between 0 and 76 . The scale has high internal validity in student samples, Cronbach's $\alpha=.88-.97$ (Hulme, Hirsch \& Stopa, 2012; Mattick \& Clarke, 1998). Mattick \& Clark (1998) report an undergraduate mean of $19, \mathrm{SD}=10.1$. Students scoring at least one SD above average $(\geq 29)$ participated as per Hulme, Hirsch \& Stopa (2012).

Baseline Depression, Anxiety and Stress: Baseline depression, anxiety and stress was measured by the Depression Anxiety Stress Scale -21 (DASS-21) (Lovibond \& Lovibond, 1995). Participants indicated the degree to which statements 
including, "I felt down-hearted and blue" had applied to them over the past week by responding on a 4-point Likert scale scored 0-3 (never/not at all; sometimes; often; almost always; most of the time), providing scores between 0 and 63 . Henry \& Crawford (2005) report good internal consistency, $\alpha=.93$.

Working memory interference: As per Homer et al. (2016), working memory interference was generated by computerised tasks based on Andrade et al. (1997) and Boomsma (2013).

Experimental condition: In the visuospatial (EMs) task, letters (bold, $7 \mathrm{~mm}$ in height) were presented repeatedly at alternate sides of the computer screen. A background of $1.5 \mathrm{~cm}$ wide black and white stripes increased visuospatial interference. Participants responded to target letters by pressing the space bar. Two randomly positioned target letters among 18 distractor letters comprised one sequence, two sequences comprised one trial and three trials comprised one block. The entire task consisted of three blocks lasting 60 seconds each. Trial 1 of each block utilised the target letter $q$ and distractor letter $p$, trials 2 and 3 used targetdistractor letter pairings $d-p$ and n-m. Letters were presented for 300msec with a $200 \mathrm{msec}$ interstimulus interval. Participants were asked to refrain from moving their heads, thus moving only their eyes during the task to ensure visuospatial interference. A practice task comprising one target letter among six distractors preceded the main task. Participants were instructed to visualise their image throughout the task and were reminded at the beginning of each block. Participants were scored on number of correct responses (18 maximum). 
Control condition: The control task matched the visuospatial task exactly, with the exception that all letters were presented centrally against a white background thus not requiring EMs.

\subsection{Procedure}

Ethical approval was granted by the University of Plymouth Health and Human Sciences Ethics Committee. Participants scoring at least one SD above average on the social anxiety screening measure (SIAS) attended a 45 minute laboratory session. After briefing and informed consent, participants completed a computerised questionnaire in which they described their imagery and completed some questions on image phenomenology as part of a parallel qualitative investigation (see Homer \& Deeprose, 2017). Participants who reported experiencing intrusive social anxietyimages then visualised their image for 20 seconds (timed by the software). 0 n $10 \mathrm{~cm}$ VASs, they then rated its vividness ('How vivid was your image during the visualisation?') from 0 ('Not at all vivid') to 10 ('Extremely vivid') and emotionality ('How emotional did you feel whilst visualising your image?') from 0 ('Not at all emotional') to 10 ('Extremely emotional'). Using a random number generator, participants were randomly allocated to either the EMs task or the control task, which they completed while holding their image in mind. Directly after, they rated the vividness and emotionality of their image during the task. Participants then visualised their image again for 20 seconds and rated post-test vividness and emotionality. Finally, participants completed a mood reversal task in which they recalled and visualised an enjoyable social situation.

\section{Results}


Assumptions of parametric tests were checked and no causes for concern were identified. One extreme outlier was removed, leaving 14 participants in the EMs condition and 12 in the control condition.

\subsection{Screening, baseline and control measures}

The mean SIAS score was $40.85, \mathrm{SD}=9.43$, range $=29-58$. The mean DASS-21 score was $20.58, S D=9.60$, range $=5-40$, and the scale showed good internal consistency, $\alpha=.89$. Independent samples $t$ tests showed no differences between groups in SIAS or DASS-21 scores, ps > .304.

Participants who completed the control task, $M=17.42, S D=.79$, range $=16-18$, were significantly more accurate in their responses than those who completed the EMs task, $\mathrm{M}=16.71, \mathrm{SD}=.83$, range $=15-18, t(24)=-2.20, p=.037, d=-0.87$.

\subsection{Self-rated Image Vividness}

Baseline vividness scores were significantly higher in the EMs condition, $t(24)=$ 2.50, $p=.02, d=0.98$, and so scores were standardised by dividing participants' baseline, during-task, and post-test vividness scores by their baseline vividness scores across both conditions. A 3×2 mixed ANOVA revealed significant main effects for both time, $F(2,48)=11.80, p<.001, \eta^{2}=.30$, and condition, $F(1,24)=5.82, p=$ $.024, \eta^{2}=.20$, and a significant interaction, $F(2,48)=3.35, p=.043, \eta^{2}=.09$ (see fig. 1). Descriptive statistics are shown in table 1.

Table 1 
Mean raw and standardised scores for vividness and mean scores for emotionality. SDs and ranges shown in brackets.

\section{Eye movements}

$n=14$

\section{Control}

$\mathrm{n}=12$

\section{Vividness}

\section{Raw scores}

Baseline

During task

Post-test visualisation

Standardised scores

Baseline

During task

Post-test visualisation
7.36

$(S D=1.69$, range $=4-10)$

3.79

$(\mathrm{SD}=2.26$, range $=0-7)$

6.50

$(S D=1.95$, range $=3-10)$

1.00

$(\mathrm{SD}=0$, range $=1-1)$

.57

$(\mathrm{SD}=.36$, range $=0-1.17)$

.90

$(\mathrm{SD}=.25$, range $=.43-1.29)$
5.33

$(S D=2.42$, range $=2-9)$

3.50

$(\mathrm{SD}=1.98$, range $=1-7)$

6.42

$(S D=1.56$, range $=4-9)$

1.00

$(\mathrm{SD}=0$, range $=1-1)$ .76

$(\mathrm{SD}=.53$, range $=.11-2)$ 1.45

$(\mathrm{SD}=.76$, range $=.83-3.5)$

\section{Emotionality}

Baseline

5.57

5.92

During task

$(\mathrm{SD}=1.45$, range $=3-8)$

3.86

$(S D=2.02$, range $=3-10)$

2.75

Post-test visualisation

$(\mathrm{SD}=2.07$, range $=0-7)$

4.93

$(\mathrm{SD}=1.76$, range $=0-6)$

$(\mathrm{SD}=1.94$, range $=0-7)$

$(\mathrm{SD}=1.50$, range $=3-8)$ 


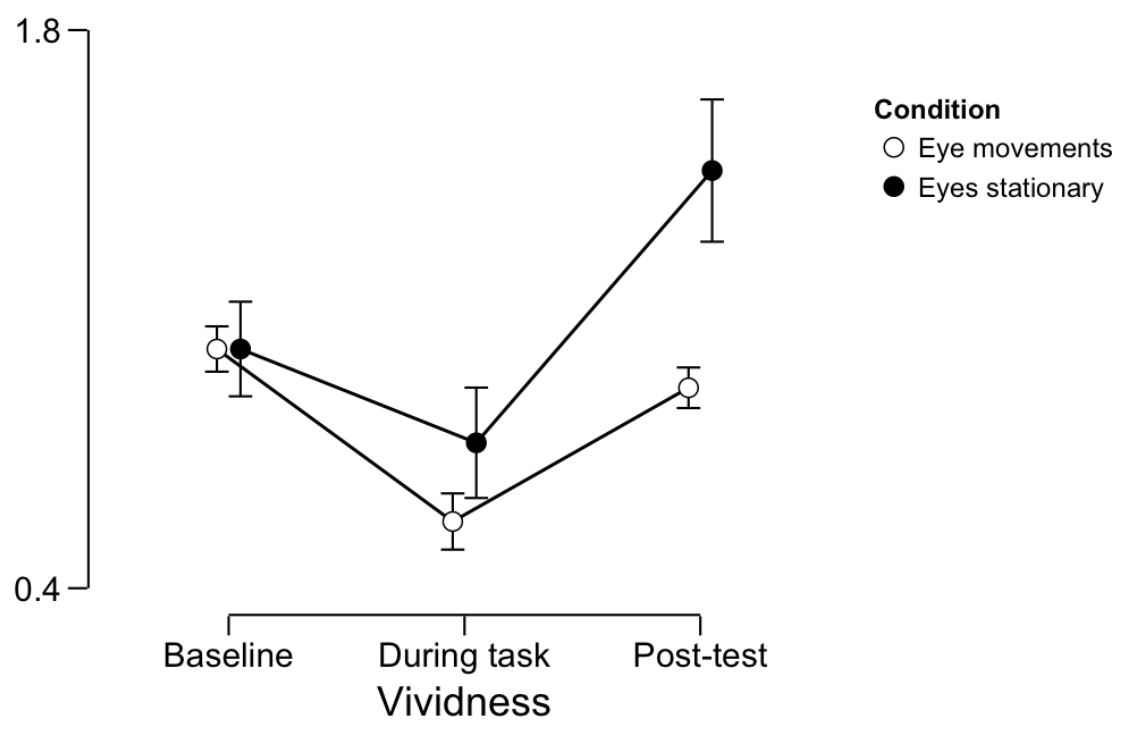

Figure 1: Vividness scores at baseline, during the task and at the post-test visualisation for both conditions.

Paired and independent samples $t$ tests showed that while there were no betweengroup differences in vividness during the task, $t(24)=-1.13, p=.269$, vividness had reduced significantly from baseline in the EMs condition, $t(13)=4.51, p=.001, d=$ 1.21, but not the control condition, $t(11)=1.55, p=.150$. Vividness increased from during the task to post-test in both conditions, $t(13)=-3.77, p=.002, d=-1.01$ (EMs), $t(11)=-2.82, p=.017, d=-0.81$ (control), but was significantly higher at posttest in the control condition, $t(13.02)=-2.37, p=.034, d=.97$ (equal variances not assumed). Neither the drop in vividness from baseline to post-test in the EMs condition nor the increase in vividness from baseline to post-test in the control condition reached significance, $t(13)=1.46, p=.169(\mathrm{EMs}), t(11)=-2.03, p=.067$ (control). 


\subsection{Self-rated Image Emotionality}

An independent samples $t$ test showed no differences in baseline emotionality scores between groups, $t(24)=-.51, p=.618$. A $3 \times 2$ mixed ANOVA revealed a significant main effect for time, $F(2,48)=22.62, p<.001, \eta^{2}=.045$, but not condition, $F(2,48)=.00, p=.989$. The time $\mathrm{x}$ condition interaction showed a statistical trend but did not reach significance, $F(2,48)=3.16, p=.051, \eta^{2}=.064$. See table 1 for descriptive statistics.

Paired and independent samples $t$ tests to investigate this trend showed that during the tasks, emotionality dropped significantly from baseline in both conditions, $t(13)=3.12, p=.008, d=.84$ (EMs), $t(11)=4.30, p=.001, d=1.24$ (control), and then increased again at post-test, $t(13)=-2.38, p=.033, d=-0.64$ (EMs), $t(11)=-$ $5.00, p<.001, d=-1.44$ (control). There were no between-group differences in emotionality at either time-point, $t(24)=1.45, p=.159$ (during task), $t(24)=-1.07, p$ $=.295$ (post-test), and neither group's emotionality decreased from baseline to posttest, $t(13)=1.26, p=.229(\mathrm{EMs}), t(11)=.609, p=.555$ (control).

\section{Discussion}

As predicted, and in line with the WM framework, a concurrent EMs task reduced image vividness more so than a similar control task not requiring EMs. Vividness in the EMs condition returned to baseline levels at post-test while a statistical trend indicated an increase in vividness from baseline to post-test in the control condition. This finding does not reflect most previous research, in which post-test vividness ratings are typically lower than baseline ratings (e.g. Barrowcliff, Gray, Freeman \& MacCulloch, 2004; Engelhard et al. 2010; Kavanagh et al., 2001; Kemps \& Tiggemann, 2007; van den Hout, et al., 2001). Interestingly, a similar result was 
observed by Engelhard, van den Hout et al. (2011), who investigated the effects of EMs on intrusive prospective imagery. As observed here, the drop in vividness in the EMs condition and increase in vividness in the control condition did not reach significance, but vividness at post-test was higher in the control condition than in the EMs condition. These effects were weaker than those of a similar study using nonintrusive prospective imagery (Engelhard et al., 2010). Together, these results suggest that intrusive imagery may be more resilient to EM attenuation, but EMs may prevent increases in vividness caused by exposure. That intrusive images should behave differently under WM interference to voluntarily generated images is unsurprising given the intrinsic differences between voluntary and involuntary memories (Brewin \& Holmes, 2003; Conway \& Pleydell-Pearce, 2000; Ehlers \& Clarke, 2000; Homer \& Deeprose, 2017), but this hypothesis requires further investigation. While this distinction could be considered by practitioners, its impact on therapy is not yet clear.

Effects for emotionality did not mirror those for vividness. Emotionality dropped during the concurrent tasks in both conditions, with no differences between groups and no reductions from baseline to post-test. This is in keeping with several studies showing that effects for emotionality are not as reliable as those for vividness (e.g. Andrade et al., 1997, experiments 1 - 3; Homer et al., 2016; Leer, Engelhard \& van den Hout, 2014). Future research should use more sensitive emotionality measures, such as implicit and explicit self-esteem and state anxiety, or objective measures such as galvanic skin response. Given the high comorbidity between social anxiety and depression, future emotionality measures should seek to differentiate between the two constructs. 
The main finding, that vividness was lower after the EMs task than after the control task, has several implications. EMDR is applied to a range of disorders, but its evidence base beyond PTSD is largely anecdotal (van den Hout \& Engelhard, 2012). This study represents a tentative first step in basic science justification for EMDR in social anxiety, in that EMs may prevent increases in image vividness and therefore consumption of WM resources during exposure, thus allowing more resources to be allocated to other therapeutic procedures (see Shapiro, 1995; Shapiro and Forrest, 2001 and Stewart et al., in prep.). However, conclusions drawn from this study reflect intrusive images only - effects of WM interference on voluntarily generated, negative, episodic memories in social anxiety (e.g. for those who do not experience intrusive imagery) are not yet known.

The study has several limitations. As a small pilot study, statistical power may have been lacking and so implications are tentative. Our results are specific to a subclinical student sample: while low-level interventions for sub-clinical distress are a worthy pursuit (Homer \& Deeprose, 2017), implications for clinical samples are limited. Including a clinically-diagnosed socially anxious group would strengthen applied implications. We cannot conclude that beneficial effects of the EMs task were due to increased visuospatial WM interference specifically (e.g. Andrade et al., 1997), as the differences in task accuracy suggest that the EMs task may have required more general cognitive resources than the control task (e.g. Gunter \& Bodner, 2008; see Stewart et al., in prep. for detailed discussion). Most participants were white British, but ethnicity was not formally recorded. 
Further experimental research should replicate the study in analogues of other disorders in which intrusive imagery is problematic. Post-test measures could include social anxiety as well as image vividness and emotionality, and longitudinal investigations would provide more insight into clinical utility.

\subsection{Conclusions}

EMs may prevent increases in the vividness of intrusive social anxiety images caused by exposure. However, in line with several previous studies, EMs did not appear to have any benefits for image emotionality, suggesting that the relationship between the vividness and emotionality of mental imagery should be investigated more thoroughly. This finding provides a preliminary rationale for EMDR intervention for disorders beyond PTSD, in that the prevention of an increase in vividness during exposure to problematic mental imagery would allow more WM resources to be allocated to other therapeutic elements of EMDR.

\section{Conflict of Interest}

This research did not receive any specific grant from funding agencies in the public, commercial, or not-for-profit sectors. The authors declare that there is no conflict of interest.

\section{Acknowledgements}

The authors gratefully acknowledge Martyn Atkins of Plymouth University for developing the computer software necessary for this study, Adam Stewart for assistance with data collection, and Onshell Relf for advice on statistical analysis. 


\section{References}

Andrade, J., Kavanagh, D., \& Baddeley, A. (1997). Eye-movements and visual imagery: A working memory approach to the treatment of post-traumatic stress disorder. British Journal of Clinical Psychology, 36(2), 209-223.

Barrowcliff, A. L., Gray, N. S., Freeman, T. C., \& MacCulloch, M. J. (2004). Eyemovements reduce the vividness, emotional valence and electrodermal arousal associated with negative autobiographical memories. Journal of Forensic Psychiatry \& Psychology, 15(2), 325-345.

Bisson, J. I., Ehlers, A., Matthews, R., Pilling, S., Richards, D., \& Turner, S. (2007). Psychological Treatments for chronic post-traumatic stress disorder: Systematic review and meta-analysis. British Journal of Psychiatry, 190, 97104.

Boomsma, C. (2013). Visual Images as a Motivational Bridge to Pro-Environmental Behaviour: A Cognitive Approach. (Doctoral dissertation). Retrieved from the Plymouth Electronic Archive and Research Library: http://hdl.handle.net/10026.1/1546

Brewin, C. R., Gregory, J. D., Lipton, M., \& Burgess, N. (2010). Intrusive Images in Psychological Disorders: Characteristics, Neural Mechanisms and Treatment Implications. Psychological Review, 117(1), 210-232.

Brewin, C. R. and Holmes, E. A. (2003). Psychological theories of post-traumatic stress disorder. Clinical Psychology Review, 23, 339-376. 
Clark, I. A., James, E. L., lyadurai, L., \& Holmes, E. A. (2015). Mental imagery in psychopathology: from the lab to the clinic. In L. A. Watson \& D. Bernsten (Eds.). Clinical Perspectives on Autobiographical Memory. Cambridge University Press.

Cloitre, M. (2009). Effective psychotherapies for posttraumatic stress disorder: A review and critique. CNS Spectrum, 14(1), 32-43.

Conway, M. A. and Pleydell-Pearce, C. W. (2000). The construction of autobiographical memories in the self-memory system. Psychological Review, 107, 261.

Ehlers, A. and Clark, D. M. (2000). A cognitive model of posttraumatic stress disorder. Behaviour Research and Therapy, 38, 319-345.

Engelhard, I. M., Sijbr, M., van den Hout, M. A., \& Rutherford, N. M. (2011). Choking under pressure: Degrading flashforwards related to performance anxiety. In http://dx. doi. org/10.5127/jep. 024111 Journal of Experimental Psychopathology, Volume 3 (2012), Issue 5, 724-738 736.

Engelhard, I. M., van den Hout, M. A., Dek, E. C., Giele, C. L., van der Wielen, J. W., Reijnen, M. J., \& van Roij, B. (2011). Reducing vividness and emotional intensity of recurrent "flashforwards" by taxing working memory: An analogue study. Journal of anxiety disorders, 25(4), 599-603. 
Engelhard, I. M., van den Hout, M. A., Janssen, W. C., \& van der Beek, J. (2010). Eye movements reduce vividness and emotionality of "flashforwards", Behaviour Research and Therapy, 48, 442-447.

Gunter, R. W., \& Bodner, G. E. (2008). How eye movements affect unpleasant memories: support for a working memory account. Behaviour Research and Therapy, 46, 913-931.

Hackmann, A., Clark, D. M., \& McManus, F. (2000). Recurrent images and early memories in social phobia. Behaviour Research and Therapy, 38(6), 601-610.

Hackmann, A., Ehlers, A., Speckens, A., \& Clark, D. (2004). Characteristics and Content of Intrusive Memories in PTSD and Their Changes With Treatment. Journal of Traumatic Stress, 17(3), 231-240.

Hackmann, A., \& Holmes, E. A. (2004). Reflecting on imagery: A clinical perspective and overview of the special issue of Memory on mental imagery and memory in psychopathology. Memory, 12(4), 389-402.

Henry, J. D., \& Crawford, J. R. (2005). The short form version of the Depression Anxiety Stress Scales (DASS-21): Construct validity and normative data in a large non-clinical sample. British Journal of Clinical Psychology, 44, 227-239.

Hirsch, C. R., Clark, D. M., Mathews, A., \& Williams, R. (2003). Self-images play a causal role in social phobia. Behaviour research and therapy, 41(8), 909-921. 
Hirsch, C., Meynen, T., \& Clark, D. (2004). Negative self-imagery in social anxiety contaminates social interactions. Memory, 12(4), 496-506.

Holmes, E. A., Grey, N., \& Young, K. A. D. (2004). Intrusive images and "hotspots of trauma memories in Posttraumatic Stress Disorder: an exploratory investigation of emotions and cognitive themes. Journal of Behaviour Therapy and Experimental Psychiatry, 36(1), 3-17.

Homer, S. R., \& Deeprose, C. (2017). Voluntary and Involuntary Imagery in Social Anxiety. Behavioural and Cognitive Psychotherapy, 45(3), 285-299.

Homer, S. R., Deeprose, C., \& Andrade, J. (2016). Negative mental imagery in public speaking anxiety: Forming cognitive resistance by taxing visuospatial working memory. Journal of behavior therapy and experimental psychiatry, 50, 77-82.

Hulme, N., Hirsch, C., \& Stopa, L. (2012). Images of the Self and Self-Esteem: Do Positive Self-Images Improve Self-Esteem in Social Anxiety? Cognitive Behaviour Therapy, 41(2), 163-173.

Jeffries, F. W., \& Davis, P. (2013). What is the role of eye movements in eye movement desensitization and reprocessing (EMDR) for post-traumatic stress disorder (PTSD)? A review. Behavioural and cognitive psychotherapy, 41(03), 290-300.

Kavanagh, D. J., Freese, S., Andrade, J., \& May, J. (2001). Effects of visuospatial tasks of desensitization to emotive memories. British Journal of Clinical Psychology, 40, 267-280. 
Kearns, M., \& Engelhard, I. M. (2015). Psychophysiological responsivity to scriptdriven imagery: An exploratory study of the effects of eye movements on public speaking flashforwards. Frontiers in psychiatry, 6, 115.

Kemps, E., \& Tiggemann, M. (2007). Reducing the vividness and emotional impact of distressing autobiographical memories: the importance of modality specific interference. Memory, 15, 412-422.

Kemps, E., Tiggemann, M., Woods, D., \& Soekov, B. (2004). Reduction of food cravings through concurrent visuospatial processing. International Journal of Eating Disorders, 36(1), 31-40.

Lee, C. W., \& Cuijpers, P. (2013). A meta-analysis of the contribution of eye movements in processing emotional memories. Journal of Behaviour Therapy and Experimental Psychiatry, 44(2), 231-239.

Leer, A., Engelhard, I. M., \& van den Hout, M. A. (2014). How eye movements in EMDR work: changes in memory vividness and emotionality. Journal of behavior therapy and experimental psychiatry, 45(3), 396-401.

Lilley, S. A., Andrade, J., Turpin, G., Sabin-Farrell, R., \& Holmes, E. A. (2009). Visuospatial working memory interference with recollections of trauma. British Journal of Clinical Psychology, 48, 309-321. 
Lovibond, P. F., \& Lovibond, S. H. (1995). The structure of negative emotional states: Comparison of the Depression Anxiety Stress Scales (DASS) with the Beck Depression and Anxiety Inventories. Behaviour Research and Therapy, 33, 335-343.

Mattick, R. P., \& Clark, J. C. (1998). Development and validation of measures of social phobia scrutiny fear and social interaction anxiety. Behaviour Research and Therapy, 36(4), 455-470.

McClelland, A., Kemps, E., \& Tiggemann, M. (2006). Reduction of vividness and associated craving in personalized food imagery. Journal of Clinical Psychology, 62(3), 355-365.

Moscovitch,D. A., Gavric, D. L.,Merrifield, C., Bielak, T., \& Moscovitch, M. (2011). Retrieval properties of negative vs. positive mental images and autobiographical memories in social anxiety: Outcomes with a new measure. Behaviour Research and Therapy, 49(8), 505-517.

National Institute for Clinical Excellence (2005). Post-Traumatic Stress Disorder (PTSD): The management of PTSD in adults and children in primary and secondary care. NICE Guidelines. Retrieved from: https://www.nice.org.uk/guidance/cg26

Ng, A. S., Abbott, M. J., \& Hunt, C. (2014). The effect of self-imagery on symptoms and processes in social anxiety: A systematic review. Clinical psychology review, 34(8), 620-633. 
Shapiro, F. (1995). Eye movement desensitization and reprocessing. Basic principles, protocols, and procedures. New York: Guilford Press.

Shapiro, F., \& Forrest, M. S. (2001). EMDR: Eye movement desensitization and reprocessing. New York: Guilford.

Stewart, A., Homer, S. R., Deeprose, C. \& Andrade, J. (in prep.). Bilateral Stimulation in EMDR: A Review of Key Theories, Evidence, and Emerging Areas of Research.

van den Hout, M. A., \& Engelhard, I. M. (2012). How does EMDR work?. Journal of Experimental Psychopathology, 3(5), 724-738.

Van den Hout, M., Muris, P., Salemink, E., \& Kindt, M. (2001). Autobiographical memories become less vivid and emotional after eye movements. British Journal of Clinical Psychology, 40, 121-130. 